\title{
Calidad de gestión administrativa financiera en las municipalidades, 2020
}

\author{
Carlos Miguel Aguilar Saldaña \\ agua0122@hotmail.com \\ Gabriela del Pilar Palomino Alvarado \\ gabpal11@hotmail.com \\ Hector Manuel Suarez Rios \\ msuarez2004@hotmail.com \\ Escuela de post grado \\ Universidad César Vallejo
}

\section{RESUMEN}

El presenta trabajo de investigación está caracteriza principalmente en mejorar la gestión de la administración financiera en las municipalidades través de un adecuado control interno. La justificación armoniza con las aseveraciones de Montalvo, L. (2015), que la Administración Financiera está basado especialmente sobre el dinero, como la principal variable de las organizaciones en busca de constantes beneficios. Aquí se establece y se pone en práctica estudios y análisis de los problemas de: La inversión, El financiamiento y La administración de los activos. El objetivo es determinar un modelo de control interno para mejorar la gestión de administración financiera de la Municipalidad. Presenta un estudio de investigación aplicada y el diseño no experimental, descriptivo de corte transversal simple; como también es propositivo. Como técnica de recolección de datos se utilizó la encuesta por cuestionario, ya que permitió evaluar la dinámica y la aplicación de las normas. Finalmente, se logró determinar que la administración de inversiones, financiamiento y activos, existe significante que cubrir con un adecuado control interno. Palabras clave: Administración, finanzas, gasto, control interno 


\title{
Quality of financial administrative management in municipalities, 2020
}

\begin{abstract}
The present research work is characterized mainly in improving the management of financial administration in municipalities through adequate internal control. The justification harmonizes with the assertions of Montalvo, L. (2015), that Financial Management is based especially on money, as the main variable of organizations in search of constant benefits. Here, studies and analysis of the problems of: investment, financing and asset management are established and put into practice. The objective is to determine an internal control model to improve the financial administration management of the Municipality. It presents an applied research study and a non-experimental, descriptive, simple cross-sectional design; as it is also purposeful. As a data collection technique, the questionnaire survey was used, since it allowed evaluating the dynamics and application of the norms. Finally, it was determined that the management of investments, financing and assets, there is a significant element to cover with adequate internal control.
\end{abstract}

Keywords: Administration; finance, spending, internal control.

Artículo recibido: 25 oct. 2020 Aceptado para publicación: 07 nov. 2020 Correspondencia: agua0122@hotmail.com, Conflictos de Interés: Ninguna que declarar 


\section{INTRODUCCIÓN}

Las finanzas internacionales están establecidas por el manejo del dinero que traspasan fronteras a través de grandes economías internacionales y finanzas corporativas. Estos organismos internacionales de crédito, manejan inversión para ser colocados en los proyectos y políticas de gobierno de cada país y controlar los diferentes mercados internacionales. Es a través de la descentralización económica de los gobiernos regionales y locales se ejecuta gasto público con la responsabilidad de resguardar el dinero, distribuir de acuerdo a las necesidades y planificación. Todo esto se da mediante la eficiente aplicación del sistema integrado de administración financiera que admite localizar, desterrar acciones anómalas y establecer posturas correctivas por parte de entidad de control y fiscalización. Pese a esto, la gestión financiera es deficiente ya que existe un comportamiento irresponsable, desordenado y desmesurado del gasto de los recursos que supera el ingreso directamente recaudado y las transferencias del Foncomún. La gestión administrativa financiera es ineficiente por el débil control en sus procesos y por el poco reajuste direccionado sobre la gestión por resultados. A esto se suma, el recurso humano que trabaja en el área de la administrativa financiera; han sido contratado sin concurso meritocràtico, tuvieron en cuenta solo decisiones políticas. En tal sentido, las malas acciones dentro de la gestión de recursos financiera como la corrupción, desvío de fondos, incumplimiento de metas, mala planificación presupuestal, personal inadecuado, mala toma de decisiones; han hecho que los fondos no se invierte en obras de impacto social en beneficio de la comunidad, buscando el bienestar socioeconómico, desarrollo y crecimiento.

El tema de estudio se sustenta con las teorías aportadas por los diferentes autores sobre la administración financiera. Así tenemos, Van, J. (2002) que define como la adquisición, el financiamiento y la administración de los activos, con el propósito de manejo económico ideal. Por consiguiente, la función se orienta a la toma de decisiones y fraccionada en espacios importantes como: inversión, financiamiento y la administración de los activos. Para que sea eficiente se requiere de cumplimiento de metas u objetivos y medir sobre criterios de eficiencia y eficacia.

De igual forma, Ortega, A. (2002)- Precisa, las funciones y responsabilidades de los administradores financieros dentro de una organización, y las funciones son claves en este aspecto de la gestión, debido por el grado de responsabilidad que asume cada uno de 
ellos. Es tas funciones y responsabilidades se basan específicamente en: La inversión, el financiamiento y las decisiones. Por consiguiente, las primordiales funciones que se llegan a establecer son: planear, obtener y hacer un adecuado manejo eficientemente de los fondos. Una buena gestión Financiera contribuye a que la entidad ejecutora cumpla sus metas y a que rivalice con triunfos con otras entidades del estado en la ejecución presupuestal.

Al respecto, Montalvo, L. (2015) refiere que, la Administración Financiera está basado especialmente sobre el dinero, como la principal variable de las organizaciones en busca de constantes beneficios. Aquí se establece y se pone en práctica estudios y análisis de los problemas de: i) La inversión, ii) El financiamiento y iii). La administración de los activos. Lo que implica, el manejo de capitales para financiar pagos a los inversionistas y gestores de los activos.

\section{Dimensiones de la administración financiera}

Presenta 3 dimensiones Van, J. (2002).

\section{Inversión}

Es el más transcendental de las tres, comienza con el establecimiento del total de activos que requieren poseer. Las autoridades de jerarquía necesitan determinar que inversiones van a ser necesarios para el beneficio de la población, en base a las prioridades de obras de impacto. Es necesario tener en cuenta la gama de inversiones, el desvió o fuga de capitales, es necesario como política de la entidad reducir, eliminar o sustituir aquellos activos que conducen a pérdidas y son inviables, para no afectar la economía. La administración efectiva de inversiones de capital demanda y considera como indicadores a: estructura administrativa eficiente y eficaz como: (i) Crear propuestas de inversión. (ii) Proyectar los flujos de caja para las propuestas. (iii) Examinar minuciosamente los flujos de caja. (iv) Identificar y seleccionar proyectos nacientes de las necesidades de la población a través del presupuesto participativo. (v) Realizar seguimiento permanente y revaluación de los proyectos de inversión. El segundo como: Estimación de Flujos de Caja, como una dinámica de valorar y tomar medidas sobre el capital. Esto establece en evaluar los flujos de caja para nuevos proyectos. Puesto, el efectivo es vital para todas las decisiones, los beneficios que se espera se enuncia en flujos de caja más que utilidades. La institución invierte con el propósito de recibir luego provechos y beneficios para la 
población. Los ingresos pueden invertirse en otros proyectos y gastos operativos de la Municipalidad. Y, por último, el Flujo de Caja Proyectado,

\section{El financiamiento,}

Los administradores financieros se centran en la integración de la parte de los pasivos, se analiza la mezcla de financiamiento de las instituciones, y se observan si existen marcadas diferencias. Además, las políticas de endeudamiento pasan por aprobación del concejo municipal, como parte de las decisiones de financiamiento. Cuando se ha decidido la forma del financiamiento, se debe de establecer los protocolos de cautela de manera física los fondos, cabe precisar que hay que decretar la forma de un préstamo a corto plazo o mediano plazo; y de igual forma los apalancamientos y gestión en los diferentes niveles de gobierno. Considera como indicador el financiamiento a corto plazo.

Al cual, Flores, J. (2010), precisa que si una empresa no concibe recursos económicos para sus diligencias, apela al financiamiento a corto plazo para forjar recursos financieros a través de tres medios, los cuales son: (i) Crédito Comercial. (ii) Prestamos de entidades financieras. (iii) Títulos Valores. El Crédito Comercial es una forma de financiamiento, ya que no cobran interés expresos sobre las cuentas pendientes. Las Fuentes de financiamiento de una entidad pública se darán a través de corto, mediano y largo plazo. Las entidades financieras prestadores de este servicio son: el Banco de la nación, bancos privados. Las fuentes internas de financiamiento. Generalmente se da a través de los recursos directamente recaudados por los tributos o arbitrios que cobras las municipalidades.

\section{Administración de activos.}

Cuando se logra la posesión de activos y se consigue financiamiento es adecuado realizar una gestión de manera responsable y con eficacia. Son las personas encargadas de esta función los que ostentan una voluminosa carga de responsabilidad operativa del manejo de los activos existentes. La tarea e interacción los obliga a la delicadeza y custodia sobre el manejo de activos circulantes que por el de activos fijos. La gran responsabilidad del manejo de los activos fijos estaría ligado a los administradores operativos. Dentro de los indicadores tenemos a la Administración de efectivos. Flores, J. (2010), señala que el término Fondo se utilizará generalmente como:(i). Caja en efectivo. (ii) Recursos Financieros Totales. (iii) Capital de Trabajo, es decir el Activo Corriente restando con el Pasivo Corriente, conocido como Capital de Trabajo Neto. Por ello es conveniente 
administrar el efectivo de manera eficiente y eficaz y no caer en aprietos financieros por falta de efectivo. Los Procedimientos para administrar el efectivo se da de la siguiente manera: (i) Los cheques se deben depositar de manera inmediata. (ii) Los pagos se de ben demorar lo menos posible para no infringir en gastos financieros. (iii). Control eficiente de los desembolsos en efectivo, etc. Por otro lado, la Administración de las Cuentas por Cobrar está sustentado en las normas y líneas técnicas en la que se ampara el Gerente Financiero de la entidad pública, además, el propósito es de brindar modalidades y formas de cancelación a los clientes. El gerente financiero, debe tener en cuenta para el cobro de los tributos lo siguiente: (i) La reputación crediticia del cliente, (ii) Historial de pagos (iii), Periodos de pago promedio, (iv) Persona Natural (promedio de ingresos), (v) Persona Jurídica (Estados Financieros), etc. Se dispondrá de diversa Técnicas de cobranza como: (i) Notificación por escrito, (ii) Llamadas Telef6nicas, (iii) Visitas personales, (iv) Mediante agencias de cobranzas y (v) Recurso legal. Otro indicador considerado es la Administración de las cuentas por cobrar. Son directrices técnicos que utiliza con la finalidad de conceder facilidades de pago a los clientes.

La gestión de la administración financiera, según Mendoza, K. (2016), señala que, es las responsabilidades de llevar a cabo sobre un proceso de actividades, esto puede ser empresarial, personal o pública, lo que incluye: (i) La inquietud por la disposición de los recursos y estructuras. (ii) La conexión de sus actividades, y sus semejantes.

Las fuentes de financiamiento, estudia las tendencias del mercado global para avizorar el rumbo y de continuar avanzando y de realizar su planificación de manera estratégica su presupuesto partiendo de las oportunidades que se presentan; de igual forma, indaga $\mathbf{y}$ analiza el nivel de riesgo de dinámica financiero de la organización. Los objetivos son: adquirir recursos para el buen ejercicio, alcanzar el uso adecuado de manera eficiente de recursos financieros establecidos, menguar de manera responsable la inseguridad y peligros a la hora de tomar decisiones, ampliar las beneficios económicos y el patrimonio neto, mediante criterios sustentables como la reducción de costos o exclusión de gastos superfluos, proveer información para una responsable y adecuada toma de decisiones. Del mismo modo, El Ministerio de Economía y Finanzas según Ley 28411, establece que el PIA; como presupuesto de inicio que tiene una entidad del estado, aprobado por el equipo de gestión, conformada por la plana directriz a cargo 
de los créditos presupuestales determinados en el Presupuesto de Sector Público para el año fiscal siguiente. En este caso los niveles de gobiernos locales y regionales. Los créditos presupuestales se aprueban primero en el consejo municipal y regional; luego se realiza el acto administrativo a través de resoluciones. Presupuesto Institucional Modificado, es a consecuencia de otras modificatorias de específicas, generalmente a nivel institucional como funcional programático, ejecutado en el presente año fiscal, a partir del PIA.

Control Interno. Constitución Política del Perù (1993). Capítulo IV. Del Régimen Tributario y Presupuestal. Artículo 82 (p.12). Control Gubernamental. Congreso de la república Ley $\mathrm{N}^{\mathrm{o}} 27785$. Capitulo II artículo 6 (2002, p. 226885). Ley $\mathrm{N}^{\mathrm{a}}$ 28716. Ley de control interno de las entidades del estado. Norma de Contraloría RC No 320 -2006-CG. Guía para la Implementación del Sistema de Control Interno de las Entidades del Estado. RC. No 458-2008-CG. Ejercicio del Control Preventivo por CGR y OCI. RC No 119-2012. CG. Ley 29743 que modifica el art. 10 de la Ley 28716. R.S.G 045-2020-CG-SGE. Legitimización del Centro de Operaciones de Control, de manera que centralice la información producto del control gubernamental a nivel nacional, con el propósito de monitorear persistentemente las acciones y disposiciones amparadas, dentro de la Emergencia Sanitaria por el COVID-19.R.C 102-2020-CG. Establece uso forzoso de "Plataforma para la Transparencia de la Gestión Pública en la Emergencia Sanitaria COVID-19", por parte de los Gobiernos Locales. R.C 100-2020-CG Adicionan la Sexta Disposición Complementaria Final a la Directiva N ${ }^{\circ}$ 002-2019CG/NORM Servicio de Control Simultáneo, en asuntos de Estado de Emergencia. Ley del Servicio Civil No 30057. Título VI. Régimen disciplinario y procedimiento sancionador. De igual forma, (Koontz y Weihrich, (2013). Señala que, el control es aquel proceso que supervisa las diligencias para aseverar que todo lo establecido en el plan se ejecute, corrigiendo de manera oportuna y en el acto cualquier mal manejo o desorientación. De la misma manera, Gámez, I. (2018) Conceptualiza a la Auditoria de la siguiente forma, proceso sistemático relacionado a alcanzar y evaluar imparcialmente documentos sobre financiamientos de carácter monetario; con el fin de establecer el nivel de responsabilidad, para luego comunicar de manera oportuna y pertinente los resultados a los interesados. También aporta, Rom -Re, A. (2017). Manifiesta que la auditoria es el análisis de los registros que sustentan las operaciones que realiza en forma habitual 
a una fecha establecida, realizado de manera práctica por el auditor para enunciar informe de auditoría, que permita establecer objetivamente el cumplimiento de manera eficiente y eficaz los objetivos y metas establecidas en la planificación a través de los planes operativos y sobrelleve a efectuar de manera objetiva comentarios, conclusiones y recomendaciones en la búsqueda de la mejora de los procesos relacionados con la gestión financiera y el control interno.

Como también, Contraloría General de la república (2014) define al Control Interno como una acción completa realizado por el gerente, director o representante de la entidad, funcionario y servidores, trazado para afrontar los peligros y establecer seguridad, direccionado al cumplimiento de la misión y los objetivos; es decir, encauzada a menguar los riesgos.

\section{MATERIALES Y MÉTODOS.}

La investigación utilizó el método lógico deductivo consistente en la aplicación de los principios teóricos sobre la Gestión de la administración financiera para analizar y encontrar los puntos críticos de las dimensiones de inversión, financiamiento y administración de activos en la Municipalidad. La población estaba conformada por todos los trabajadores de la municipal. La muestra estuvo conformada por 19 trabajadores, 4 del área de administración, 4 presupuesto, 3 contabilidad, 3 abastecimiento, 5 rentas. Se utilizó el cuestionario sobre aspectos de la gestión de la administración financiera, inversiones y activos. En cuanto al sistema de control se tomó en cuenta las siguientes dimensiones: ambiente de control, evaluación de riesgos, actividades de control, información y comunicación y supervisión. Para el grado de confiabilidad se utilizó el Alfa de Cronbach.

\section{RESULTADOS Y DISCUSIÓN}

A continuación se expone los resultados adquiridos fruto de una minuciosa y responsable aplicación del instrumentos de investigación, el cuestionario; se aplicaron a los colaboradores de las diversas áreas como presupuesto, contabilidad, abastecimiento, rentas, comprometidas con la gestión de la administración financiera de la Municipalidad, así también se realizó análisis y contrastación en la medida de las posibilidades e interés de toda la información. Se tuvo en cuenta siempre el horizonte de los objetivos trazados con imparcialidad para los resultados de la investigación. 
Tabla 1. Administración de inversiones de la municipalidad

\begin{tabular}{|c|c|c|c|c|c|c|c|}
\hline \multicolumn{2}{|c|}{ Administración de inversiones } & \multicolumn{2}{|c|}{$\begin{array}{l}\text { Nunca/ } \\
\text { Casi } \\
\text { nunca }\end{array}$} & \multicolumn{2}{|c|}{$\begin{array}{l}\text { Algunas } \\
\text { veces }\end{array}$} & \multicolumn{2}{|c|}{$\begin{array}{c}\text { Casi } \\
\text { siempre/ } \\
\text { Siempre }\end{array}$} \\
\hline & & $\mathrm{Fi}$ & $\%$ & fi & $\%$ & fi & $\%$ \\
\hline \multirow{4}{*}{$\begin{array}{c}\text { Estructura } \\
\text { administrativa. }\end{array}$} & $\begin{array}{l}\text { Proyección de los flujos de caja para } \\
\text { las propuestas de inversión }\end{array}$ & 18 & $94.7 \%$ & 1 & $5.3 \%$ & 0 & $0.0 \%$ \\
\hline & Evaluación de los flujos de caja & 14 & $73.7 \%$ & 5 & $26.3 \%$ & 0 & $0.0 \%$ \\
\hline & $\begin{array}{l}\text { Participación en la identificación y } \\
\text { selección de proyectos de inversión }\end{array}$ & 18 & $94.7 \%$ & 1 & $5.3 \%$ & 0 & $0.0 \%$ \\
\hline & $\begin{array}{l}\text { Participación en el seguimiento y } \\
\text { reevaluación permanente de los } \\
\text { proyectos de inversión }\end{array}$ & 14 & $73.7 \%$ & 2 & $10.5 \%$ & 3 & $\begin{array}{c}15.8 \\
\%\end{array}$ \\
\hline \multirow{2}{*}{$\begin{array}{l}\text { Estimación del } \\
\text { flujo de caja. }\end{array}$} & $\begin{array}{l}\text { Participación en la proyección de } \\
\text { flujos de caja futuros para los } \\
\text { proyectos }\end{array}$ & 16 & $84.2 \%$ & 3 & $15.8 \%$ & 0 & $0.0 \%$ \\
\hline & $\begin{array}{l}\text { Participación en la reinversión de los } \\
\text { ingresos en otros proyectos o gastos } \\
\text { operativos }\end{array}$ & 14 & $73.7 \%$ & 5 & $26.3 \%$ & 0 & $0.0 \%$ \\
\hline \multirow{2}{*}{$\begin{array}{l}\text { Flujo de caja } \\
\text { proyectado. }\end{array}$} & $\begin{array}{l}\text { Conocimiento de la entrada y salida } \\
\text { de liquidez de la institución }\end{array}$ & 13 & $68.4 \%$ & 6 & $31.6 \%$ & 0 & $0.0 \%$ \\
\hline & $\begin{array}{l}\text { Conocimiento del saldo final del } \\
\text { flujo de caja proyectado }\end{array}$ & 11 & $57.9 \%$ & 8 & $42.1 \%$ & 0 & $0.0 \%$ \\
\hline
\end{tabular}

Fuente: Cuestionario aplicado a los colaboradores de la municipalidad provincial Mariscal Cáceres

Del 100\% (19) de los trabajadores, referente a la estructura administrativa, el 94.7\% (18) indicaron Nunca o Casi Nunca se proyectan los flujos de caja para la propuesta de inversión, ni participan en la identificación y selección de dichos proyectos, respectivamente. Asimismo, el 73,7\% (14) refieren que Nunca o Casi Nunca se evalúa los flujos de caja, ni se participa en el seguimiento y reevaluación permanente de los proyectos de inversión.

Respecto a la estimación del flujo de caja, el 84,2\% (16) considera que Nunca o Casi Nunca participan en la proyección de los flujos de caja futuros para los proyectos, el $73.7 \%$ (14) ni participan en la reinversión de los ingresos en otro proyectos o gastos operativos.

Finalmente, referente al flujo de caja proyectado el 68,4\% (13) de los colaboradores manifiestan que Nunca o Casi Nunca tienen conocimiento de la entrada y salida de liquida 
de la institución, ni conocimiento del saldo final del flujo de caja proyectado en un 57,9\% (11).

Tabla 2. Administración del financiamiento en la municipalidad

\begin{tabular}{|c|c|c|c|c|c|c|c|}
\hline \multicolumn{2}{|c|}{ Administración del financiamiento } & \multicolumn{2}{|c|}{$\begin{array}{l}\text { Nunca/casi } \\
\text { nunca }\end{array}$} & \multicolumn{2}{|c|}{$\begin{array}{l}\text { Algunas } \\
\text { veces }\end{array}$} & \multicolumn{2}{|c|}{$\begin{array}{c}\text { Casi } \\
\text { siempre/ } \\
\text { Siempre }\end{array}$} \\
\hline & & $\mathrm{Fi}$ & $\%$ & fi & $\%$ & fi & $\%$ \\
\hline \multirow{2}{*}{$\begin{array}{l}\text { Financiamiento } \\
\text { a Corto plazo. }\end{array}$} & $\begin{array}{l}\text { Conocimiento de los tipos de } \\
\text { financiamiento público y privado }\end{array}$ & 9 & $47.4 \%$ & 10 & $52.6 \%$ & 0 & $0.0 \%$ \\
\hline & $\begin{array}{l}\text { Participación en la toma de } \\
\text { decisiones según fuentes de } \\
\text { financiamiento }\end{array}$ & 12 & $\%$ & 6 & $\%$ & 1 & $5.3 \%$ \\
\hline \multirow{2}{*}{$\begin{array}{l}\text { Financiamiento } \\
\quad \text { a largo } \\
\text { mediano plazo. }\end{array}$} & $\begin{array}{l}\text { Conocimiento de pasivos o } \\
\text { compromisos financieros de la } \\
\text { municipalidad con instituciones } \\
\text { financieras }\end{array}$ & 13 & $68.4 \%$ & 6 & $35.3 \%$ & 0 & $0.0 \%$ \\
\hline & $\begin{array}{l}\text { Solvencia crediticia de la } \\
\text { institución }\end{array}$ & 14 & $73.7 \%$ & 5 & $26.3 \%$ & 0 & $0.0 \%$ \\
\hline \multirow{2}{*}{$\begin{array}{c}\text { Fuentes } \\
\text { internas de } \\
\text { financiamiento }\end{array}$} & $\begin{array}{l}\text { Uso de los recursos directamente } \\
\text { recaudados en obras públicas }\end{array}$ & 17 & $89.5 \%$ & 2 & $10.5 \%$ & 0 & $0.0 \%$ \\
\hline & $\begin{array}{l}\text { Recibió capacitación en sistema de } \\
\text { gestión presupuestal }\end{array}$ & 9 & $47.4 \%$ & 10 & $52.6 \%$ & 0 & $0.0 \%$ \\
\hline
\end{tabular}

Fuente: Cuestionario aplicado a los colaboradores de la municipalidad provincial Mariscal Cáceres.

Concerniente al financiamiento a corto plazo, el 63\% (12) indicaron Nunca o casi Nunca participa en la toma de decisiones para determinar las fuentes de financiamiento en la entidad. Asimismo el 52.6\% (10) precisaron que Algunas Veces tiene conocimiento de los tipos de financiamiento público o privado. Es importante la participación de los colaboradores para que compenetren en la toma de decisiones sobre las fuentes y tipos de financiamiento para el cumplimiento de los objetivos y metas trazadas.

Respecto al financiamiento a largo y mediano plazo, el 73.7\% considera que la institución tiene solvencia crediticia negativa con las entidades financieras; el 68.4\% (13) considera que Nunca o Casi Nunca tener conocimiento del pasivos o compromisos con instituciones financieras.

Por último, en relación a las fuentes de financiamiento, e $189.5 \%$ (14) indican que Nunca o Casi Nunca tener conocimiento sobre el uso de los recursos directamente recaudados para inversión en obras públicas; el $52.6 \%$ (10) considera que algunas veces recibió 
capacitación sobre temas de gestión presupuestal. Es necesario que los actores financieros conozcan en que se invierte los recursos.

\section{Tabla 3. Administración de activos en la municipalidad}

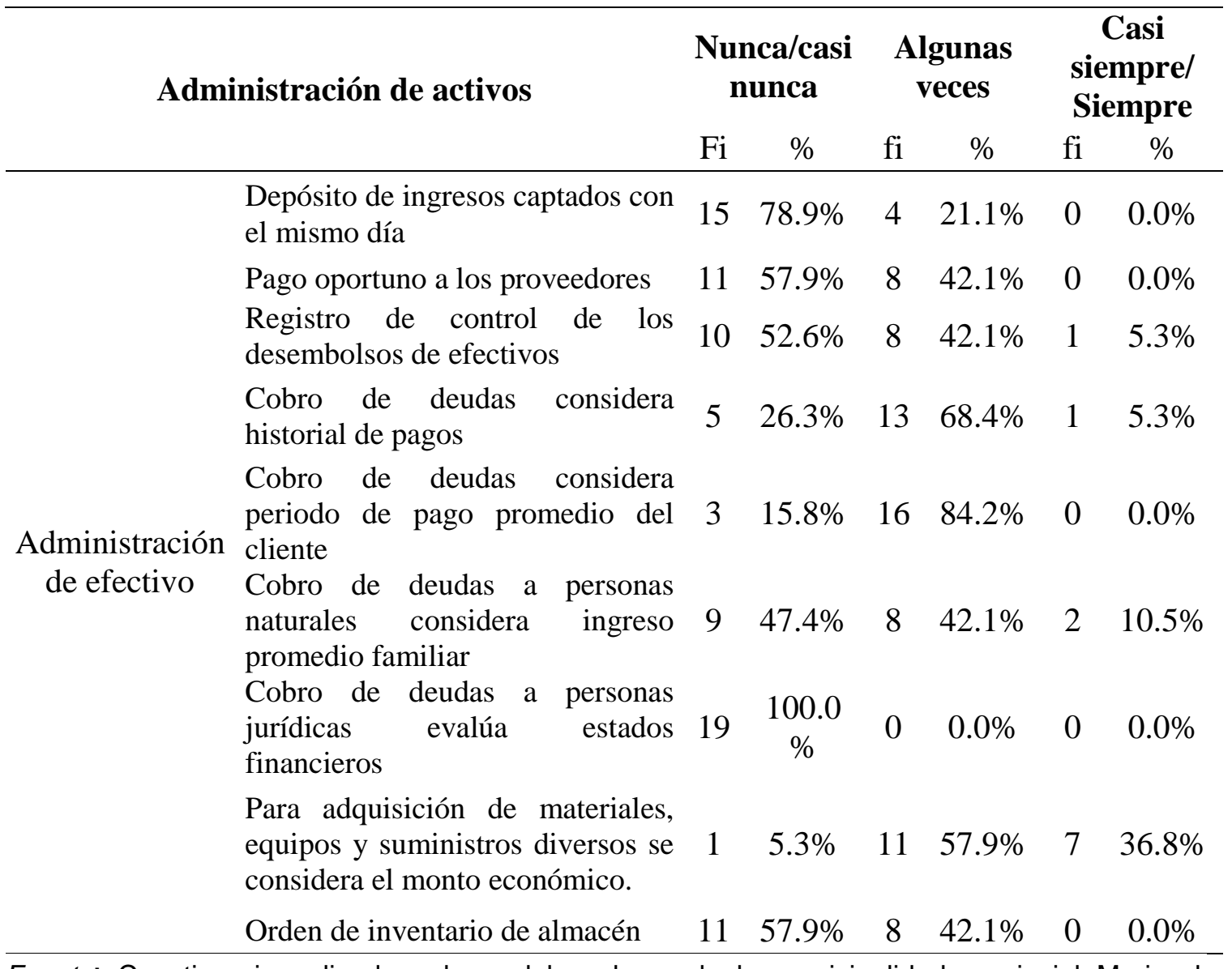

Fuente: Cuestionario aplicado a los colaboradores de la municipalidad provincial Mariscal Cáceres

En razón a la administración de efectivos, del total de encuestados el 78.9\% (15) considera que nunca o casi nunca se realiza el depósito de los ingresos captados el mismo dia; el 57.9\%(11) indicaron que nunca o casi nunca se cancela oportunamente a los proveedores, el 52\%(10) hacen referencia que Nunca o Casi nunca existe registro de control de los desembolsos de efectivos de la institución.

Al respecto de la Administración de cuentas por cobrar, el 100\%(19) indican que Nunca o Casi nunca evalúan los estado financieros para el cobro de personas jurídicas; el 84.2\% (16) considera que Algunas Veces consideran el periodo de pago promedio del cliente para el cobro de las deudas; el 68.4\%(13) manifestaron que 
algunas veces consideran el historial de pago para el cobro de las deudas; el 57.9\%/ (11) manifestaron que algunas veces se considera la reputación crediticia del cliente para el cobro de las deudas.

Finalmente, referente a la administración de inventarios, el $78.9 \%$ considera que algunas veces se registran el ingreso y salida de materiales y equipos diversos del almacén; el 57.9\% (11) manifiestan que el almacén se encuentra desordenado sin criterio de inventario y algunas veces consideran el monto económico de los de materiales, equipos y suministros diversos para la adquisición.

\section{Gestión del sistema de control interno Ambiente de control}

Concerniente al sistema organizacional; $89.5 \%$ (17) señalan que nunca o casi nunca le socializaron el plan estratégico, esto podría deberse a que, el $84.2 \%$ indican que nunca o casi nunca se actualizan ni se aprueban los instrumentos de gestión, ni participan en la elaboración del plan estratégico, tampoco cuentan con manual de procedimiento de actividades institucionales.

Referente a la asignación de responsabilidades y niveles de autoridad de control interno, el 100\% (19) indican que "Nunca o casi nunca" las auditorias se realiza en todas las áreas de la entidad, ni cuentan con personal idóneo de acuerdo al perfil de puesto (73.7\%), ni tienen autonomia para brindar información. Asimismo, el 68.4\%(13) manifiesta que los objetivos estratégicos son inconsistentes con la misión de la institución y un 63.2\% (12) afirma que "Nunca o casi nunca" las funciones de los niveles jerárquicos están establecidas en el MOF. Por otro lado, "Algunas veces" un $63,2 \%$ (12) refieren que, se relacionan los lineamientos de política institucional con las políticas nacionales y que los funcionarios toman decisiones administrativas, respectivamente.

\section{Evaluación de Riesgos}

En relación a identificación de eventos, el 68.4\% (13) manifiesta que "Nunca o casi nunca" se hizo capacitación para responder ante la ocurrencia de riesgo, ni tampoco que exista la matriz de respuesta de riesgo (57.9\%).

Así mismo, respecto a la dimensión análisis de riesgo, el 78.9\% (15) considera que "Nunca o casi nunca" existe rotación periódica de personal asignado en puestos 
susceptibles de riesgos, ni participan en el análisis de estimación de costos frente a respuestas de riesgo (73.7\%), ni el personal de la oficina de control interno contribuye en la disminución de los riesgos.

\section{Actividades de control}

Respecto a la eficiencia y eficacia de las operaciones, el 73.7\%(14) considera que "Nunca o casi nunca" se realiza revisión, análisis y adecuación de los procesos, ni elimina procedimientos innecesarios y burocráticos, tampoco se cumplen las metas establecidas en la entidad (57.9\%).

En cuenta a la confiabilidad de la información financiera, el 84.2\% (16) manifiesta que "Nunca o casi nunca" existe personal designado a generar información para evaluar la eficiencia y eficacia en la entidad; tampoco la oficina de planeamiento verifica los indicadores cuantitativos y cualitativos de la información financiera (63.2\%); pero considera que "Algunas veces" la entidad realiza modificaciones presupuestarias $(57.9 \%)$.

\section{Información y Comunicación}

En relación a la información, el $84.2 \%$ considera que "Nunca o casi nunca" existe comunicación efectiva entre las áreas de la entidad, ni canales de comunicación suficientes para sus colaboradores (el 63.2\%). Asimismo, refieren que "Algunas veces" los colaboradores muestran capacidad técnica para procesar oportunamente la información financiera $(47,4 \%)$ con tendencia a "Nunca o casi nunca" en un 42,1\%.

\section{Supervisión y Monitoreo}

Concerniente a la supervisión continua, el 94.7\% (18) considera que "Nunca o casi nunca" existe autoevaluación del proceso de control interno, ni los informes de auditoría se pone en conocimiento de la alta dirección (78.9\%), debido tal vez a que la alta dirección no promueve mecanismos efectivos para el cumplimiento de los objetivos $(68.4 \%)$.

\section{DISCUSIÓN}

La presente investigación posee como objetivo general Proponer un Control Interno para mejorar la Gestión de la Administración Financiera de la Municipalidad Provincial de Mariscal Cáceres; de lo investigado resalta que requiere de manera primordial la implementación de un control Interno, ya que existe peligro de que se 
cometan faltas graves en relación a la ejecución del gasto y/o presupuesto otorgado por el Ministerio de Economía y Finanzas, como también de los ingresos directamente recaudados y recursos producto de gestión.

En el primer objetivo específico; Analizar la administración financiera que se desarrolla en la Municipalidad; se aplicó un cuestionario que estuvo dirigido a 10 trabajadores, para la prueba de confiabilidad a través del Programa Estadístico SPSS, se obtuvo en la variable de Gestión de la administración financiera el confiabilidad del $0.7584 \%$ y en Control Interno de $0.77437 \%$. Debido a ello se puede aseverar que el cuestionario es confiable. Al aplicar esta herramienta se obtiene que la Municipalidad, en relación a la administración de inversiones; que no existe una estructura ya que ni se proyecta los flujos de caja para propuestas de inversión, ni se evalúan los flujos de caja; de igual manera, ni participan en la identificación, selección seguimiento y/o revalorización de proyecto de inversión. En relación a la estimación del flujo de caja, los colaboradores ni participan en la proyección de flujos de caja futuros para inversión en proyectos, ni en la determinación de reinversión de los ingresos en otros proyectos. Asimismo, en relación al flujo de caja proyectado, manifestaron que desconocen de la entrada y salida de liquidez y el saldo final del flujo de caja.

En una entidad es de prioridad tener en cuenta que las proyecciones, y los recursos que cuenta para determinar y priorizar las obras públicas y el cumplimiento de las metas.

Los resultados obtenidos lo comparamos con su investigación de Ortega, A. (2002) donde especifica que las funciones y responsabilidades de los administradores financieros dentro de una organización, son claves en este aspecto de la gestión, debido por el grado de responsabilidad que asume cada uno de ellos. Estas funciones y responsabilidades se basan específicamente en: La inversión, el financiamiento y las decisiones.

Desde que asumimos un cargo dentro de un área, más aún donde fluye dinero, hay que tomar con mucha responsabilidad las funciones en base a la ética y específicamente en la aplicación de las normas sobre las funciones establecidas en Manual de organización y funciones. 
Además, se evidenció que los trabajadores ni participan en la toma de decisiones para determinar la fuente de financiamiento; ni tiene conocimiento de los pasivos y compromisos, como también la institución no cuenta con solvencia crediticia y desconoce que los recursos directamente recaudados se utilizan en obras públicas. Las instituciones públicas están las áreas integradas, donde debe existir una comunicación fluida para encaminar todos al cumplimiento de los objetivos y metas. Es necesario comprender que para invertir en obras públicas, gastos operativos o determinar compromisos es necesario tener en cuenta los estados financieros de la Municipalidad para determinar una buena toma de decisiones.

Por su parte, Oliva. (2018). En su trabajo de investigación llega a la conclusión que la ejecución del gasto, es posible obtener ganancias mediante la mejora de gestión a través del análisis de sistemas administrativos, de contabilidad, tesorería, control y abastecimiento.

De igual forma, la administración de activos es inadecuado, motivo de que los ingresos captados no se depositan ese mismo día, ni se realiza el pago oportuno a los proveedores, aún más, no existe un registro de control de los desembolsos referente al manejo de efectivo; poniendo en riesgo la cautela del efectivos. Además no existen políticas adecuadas de cobranza, teniendo en cuenta la evaluación de los estados financieros de las personas jurídicas.

Asimismo, Ortega. (2002). Estipula que las funciones y responsabilidades de los administradores financieros dentro de una organización, y las funciones son claves en este aspecto de la gestión, debido al grado de responsabilidad que asume cada uno de ellos.

Es necesario precisar, una gestión financiera bien dirigida ayuda a tener una eficiente administración y capacidad de gasto en el buen uso de los recursos para cumplimento de los objetivos, de esa manera no caer en errores que conduzcan a procesos administrativas y penales.

Con los resultados obtenidos del control interno nos conduce a la imperiosa necesidad de implementar un adecuado control interno que permitiría identificar y controlar aspectos importantes de la gestión financiera, de esta menara mejorar la calidad de la gestión; y por ende del servicio en la Municipalidad. 
(Koontz y Weihrich. (2013). Manifiesta, el control es aquel proceso que supervisa las diligencias para aseverar que todo lo establecido en el plan se ejecute, corrigiendo de manera oportuna y en el acto cualquier mal manejo o desorientación.

Siendo la administración financiera un sistema integrado y delicado de mucha importancia es necesario realizar un control concurrente y corregir en el camino los aspectos necesarios para la buena marcha económica, financiera y transparente; hacia el logro de los objetivos, de manera eficaz y eficiente en el gasto público.

\section{CONCLUSIÓN O CONSIDERACIONES FINALES}

En conclusión podemos manifestar que:

1.- La Municipalidad Provincial de Mariscal Cáceres tiene una Gestión de administración financiera deficiente que superan el $70 \%$ de cumplimiento, debido a que no se proyecta, ni participan, ni se evalúan los flujos de caja. Como también, no cuenta con solvencia crediticia y los recursos directamente recaudados no se invierten en obras públicas; asimismo los ingresos captados no se depositan el mismo día lo que es un riesgo en la cautelo de los ingresos; aún más, para el cobra de las deudas no se realizan evaluación de los estados financieros para personas jurídicas.

2.- La Gestión del Control Interno en la Municipalidad Provincial, al analizar se llegó a la conclusión:

En la dimensión ambiente de control, es necesario la implementación de acciones de acceso al control y fiscalización de los recursos. Ya que el personal desconoce si los instrumentos están actualizados o aprobados, ni participan en la elaboración del plan estratégico, aún más ni lo socializan. Además el personal no cuenta con el perfil del puesto lo indica que el ambiente de control es deficiente ya que superan el $70 \%$ de incumplimiento de los indicadores.

La evaluación de riesgos, por su parte, Este elemento requiere efectuar estrategias para mejorar la participación, análisis y rotación de personal capacitado para mejorar y contribuir a la correcta ejecución de la gestión de la administración financiera.

De igual forma, el componente actividad de control, demanda de suma urgencia de estrategias para mejorar acciones que contribuyan al control, fiscalización de las 
finanzas. Tales como eliminación de procesos burocráticos, designar responsables para la evaluación de la eficiencia y eficacia.

Asimismo, Información y comunicación, requiere efectuar estrategias de manera conjunta entre todas las áreas para exista una información y comunicación efectiva, de esta manera obtener reportes pertinentes y permita una adecuada toma de decisiones.

La supervisión, se debe fortalecer mecanismos que establezcan el conocimiento oportuno del informe de auditoría, auto evaluación del proceso de control, que conduzcan a minimizar los riesgos en la Gestión Financiera.

3.- Con una eficiente, adecuada y pertinente ejecución del control interno la Municipalidad mejora significativamente la gestión financiera.

4.- Con la ejecución de la propuesta de diseño la expectativa es mejorar la de deficiencia de la administración de inversiones, financiamiento y los activos y obtener efectos favorables.

\section{LISTA DE REFERENCIAS}

Ablan, N., Méndez, Z., Los sistemas de control interno en los entes descentralizados estadales y municipales desde la perspectiva 2015. (Artículo Científico) Universidad de los Andes. Venezuela. Recuperada de https://www.redalyc.org/pdf/4655/465545883001.pdf.

Alegría, D. (2017). Human resources capacities in good practices of the financial administration system in the provincial municipality of Leoncio Prado. Huánuco Region 2016. (Scientific article), Peru. Recovered from http://revistas.unas.edu.pe/index.php/Balances/article/view/112.

Alfageme, M. y Guabloche M. (2017). Estado, Gasto Público y Desarrollo de las Capacidades: Una Aproximación. Banco Central de Reserva del Perú. Recuperado de http://www.bcrp.gob.pe/docs/Publicaciones/Revista-Estu diosEconomicos/02/Estudios-Economicos-2-4.pdf el 12 de enero del 2017. 
Apaza, M. (2010). Consultor Económico Financiero (3era edición), Perú. Instituto Pacífico, Lima, Perú.

Barcelata, H. (2019) Deuda pública subnacional y desarrollo económico local. (Artículo científico). Universidad Autónoma Metropolitana, España. Recuperada de http://www.scielo.org.mx/scielo.php?script=sci_abstract\&pid=S0188$33802019000200165 \& \operatorname{lng}=$ es \&nrm=iso.

Bautista, S. (2016). Análisis de las normas del control interno en el área de tesorería de la municipalidad distrital de Cuturapi, periodo 20016. (Artículo Científico) Universidad Nacional del Altiplano. Perú. Recuperada de http://repositorio.unap.edu.pe/bitstream/handle/UNAP/7012/ARTICULO\%20CIEN TIFICO.pdf?sequence $=3 \&$ is Allowed $=\mathrm{y}$

Brandao, M. (2018). La importancia del Control interno para prevenir el fraude en una tapicería de la industria. (Artículo Científico). Revista científica multidisciplinaria base de conocimiento. Año 03, Ed. 06, vol. 05, pp. 95-110, junio de 2018. ISSN: 2448-0959 Sao Paulo. Brasil. https://www.nucleodoconhecimento.com.br/contabilidad/prevencion-de fraude\#Resumen

Burgos, C., Suarez, R. (2017) The accounting internal control system and its influence on the financial management of the investment company CHRISTH.AL. S.A.C Trujillo district. Year 2015. (Scientific Article) Universidad Privada Antenor Orrego. Trujillo. Recovered from http://repositorio.upao.edu.pe/handle/upaorep/2354.

Cabrera, C., Fuentes, Cerezo, G. La gestión financiera aplicada a las organizaciones. (Artículo Científico)Universidad Técnica Estatal de Quevedo. Ecuador- Recuperada de http://dominiodelasciencias.com/ojs/index.php/es/index.

Chiavenato, I. (1999). Administración de Recursos Humanos (Quinta ed.). Colombia: Mc GrawHill.

Chinchilla, S. (2020) Contraloría urge a diputados reformas de fondo para lograr eficiencia en gasto. (Artículo Científico) La Nación de Costa Rica. Recuperado de https://link.gale.com/apps/doc/A619830105/IFME?u=univcv\&sid=IFME\&xid=959 dc72d. Accessed 20 Apr. 2020. 
Contraloría General de la República. (2014) Marco conceptual del Control Interno. (Revista) Perú. https://www.contraloria.gob.pe.

Contreras, L. (2018). Factores básicos para el uso de la investigación operativa como herramienta estratégica en las organizaciones. (Artículo científico). Universidad Federal de Sao Paolo, Brasil. Recuperada de

Coz, A.(2019) Rentabilidad financiera: un abordaje teórico desde la perspectiva empresarial.

(Artículo científico).Universidad Peruana Unión, Lima, Perú. Recuperada dehttps://repositorio.upeu.edu.pe/bitstream/handle/UPEU/2484/Alvaro_Trabajo_Ba chiller_2019.pdf?sequence $=1 \&$ isAllowed $=y$.

Decreto de Urgencia 014-19 Presupuesto del sector público para el año físcal 2020.

https://www.mef.gob.pe/es/por-instrumento/decreto-de-urgencia/21192-decreto-deurgencia-n-014-2019-1/file

Delfini, M. (2018). Pagos por rendimiento en las filiales argentinas de empresas multinacionales. (Artículo Científico) Universidad Nacional de la Plata, Argentina.

Gamboa, J. (2017) La planificación financiera en la mejora de la gestión administrativa en las facultades de las universidades públicas (Tesis doctoral) Universidad Estatal de Milagro Ecuador. Recuperada de https://cybertesis.unmsm.edu.pe/bitstream/handle/20.500.12672/6613/Gamboa_pj.pd f? sequence $=1$

General Comptroller of the Republic. (2014) Conceptual framework of Internal Control. (Magazine) Perú. https://www.contraloria.gob.pe.

Huacchillo, L., Ramos, M,; Pulache, J. (2020) La gestión financiera y su incidencia en la toma de decisiones financieras. (Artículo Científico) Universidad César Vallejo. Piura. Perú. Recuperada de http://scielo.sld.cu/scielo.php?script=sci_arttext\&pid=S221836202020000200356.

Khoury, F. Contraloría General de la República (2014) Marco conceptual del Control Interno. (Revista Oficial). Lima Perú https://apps.contraloria.gob.pe/wcm/control_interno/documentos/Publicaciones/Marc o_Conceptual_Control_Interno_CGR.pdf. 
Ley 28411. (2004). Ley General del Sistema Nacional de Presupuesto. Perú. Recuperado de https://cdn.www.gob.pe/uploads/document/file/255721/229463_file2018121816260-1twi55b.pdf.

Ley $N^{\circ}$ 27785. (2002) Ley Orgánica del Sistema nacional de Control y de la Contraloría general de la república. Diario Oficial el Peruano. 23 de julio 2002. Recuperada de http://www.leyes.congreso.gob.pe/Documentos/Leyes/27785.pdf

Ley $\mathrm{N}^{\circ}$ 28716. (2006). Ley de control interno de las Entidades del Estado. Diario Oficial el Peruano. 18 de abril 2006. Pág. 316871. Recuperada de http://www.leyes.congreso.gob.pe/Documentos/Leyes/28716.pdf.

Ley $N^{\circ}$ 30970. (2019) Ley General del Sistema Nacional de Presupuesto. Diario oficial el peruano. https://www.mef.gob.pe/es/por-instrumento/ley/20334-ley-n-30970/file.

Lozano, G., Tenorio, J. (2015) The Internal control system: A tool for the improvement of business management in the Construction sector. (Scientific Article) UPeU.Tarapoto. Peru. Recovered from file://C:/Users/Tula_1/Downloads/896Texto\%20del\%20art\%C3\%ADculo-1195-110-20180524.pdf

Mendoza, W., García., Delgado, M., Barreiro, I. Internal control and its influence on the administrative management of the public sector. (Scientific Article) State University of the South of Manabí. Ecuador. Recovered from DialnetElControlInternoYSuInfluenciaEnLaGestionAdministra-6656251.pdf.

Ministerio de Economía y Finanzas (2017). Presupuesto por resultados: conceptos y líneas de acción. Lima, Perú

Montalvo, L. (2015) Administración financiera básica en el corto plazo. (Tesis Doctoral) Universidad Mayor de San Andrés Facultad Ciencias Económicas y Financieras. Bolivia. Recuperado de https://repositorio.umsa.bo/bitstream/handle/123456789/7319/122645.pdf?sequence $=1 \&$ isAllowed $=\mathrm{y}$

Oliva, C. (2018). Gestión de las Finanzas Públicas en el Perú. Informe de sistematización de las evaluaciones bajo metodología PEFA realizadas a 10 gobiernos subnacionales. (Artículo científico) Lima Perú. Recuperada de 
https://www.baselgovernance.org/publications/gestion-de-las-finanzas-publicas-enel-peru.

Ortega, A., Padilla, S., Torres, J., Ruz, A. (2017). Nivel de importancia del control interno de los inventarios dentro del marco conceptual de una empresa. (Artículo Científico) Universidad Simón Bolívar. Colombia. Recuperada de http://Downloads/3261Texto\%20del\%20artículo-5773-1-10-20181016.pdf.

Pacheco, R; Robles, C.; Ospino, A. (2018) Análisis de la Gestión Administrativa en las Instituciones Educativas de los Niveles de Básica y Media en las Zonas Rurales de Santa Marta. (Artículo Científico) Universidad de Magdalena. Colombia. Recuperada de https://scielo.conicyt.cl/scielo.php?script=sci_arttext\&pid=S071807642018000500259 .

Paiva, F. (2013). Internal control and its impact on the profitability of the company cobros del norte S.A. (Scientific Article) UNAN-Managua. FAREM Nicaragua. Recovered from https://repositorio.unan.edu.ni/5910/3/99-371-1-PB.pdf

Philippatos, G C. (2020) Fundamentos de Administración Financiera, Textos y Casos, (4ra.Ed.) México. Edit. Mc Graw Hill.

Ramírez, A., Calderón, E. (2017) Administrative management in business development (Scientific Article) Escuela Superior Politécnica de Chimborazo, Ecuador. Recovered from https://www.eumed.net/ce/2017/1/gestion.html

Recuperada de https://revistas.unlp.edu.ar/CADM/article/view/4343.

Resolución de Contraloría. N ${ }^{\circ}$ 100-2020-CG. Control Simultáneo. 28 de marzo, 2020.

Diario Oficial el Peruano. https://busquedas.elperuano.pe/normaslegales/adicionansexta-disposicion-complementaria-final-a-la-direct-resolucion-n-100-2020-cg$1865210-1 /$

Resolución de Contraloría. N 102-2020-CG. Disponen el uso obligatorio de la "Plataforma para la Transparencia de la Gestión Pública en la Emergencia Sanitaria COVID-19", por parte de los Gobiernos Locales. 2 de abril del 2020. Recuperada de https://www.gob.pe/institucion/contraloria/normas-legales/606115-r-c-102-2020-cg. 
Resolución de Secretaría General Nº R.S.G 045-2020-CG. 08 de abril, 2020. Recuperada de https://www.gob.pe/institucion/contraloria/normas-legales/606112-r-s-g-045-2020cg-sge.

Sánchez, G., Taboada, D. (2015) Design and Proposal of an Internal Administrative, Financial Control System based on the Coso Erm Model, to improve the identification and analysis of risks, applied to the Corporate Group Mary Carmen, Cantón Ambato, Tungurahua Province. (Scientific Article) University of the Armed Forces. Ecuador. Recovered from https://repositorio.espe.edu.ec/bitstream/21000/10019/1/AC-ESPELCAI-0431A.pdf

Terrazas, R. (2009) Modelo de gestión financiera para una organización.(Artículo Científico) Universidad Católica Boliviana San Pablo. Bolivia. Recuperada de https://www.redalyc.org/pdf/4259/425942159005.pdf

Valera, J. (2019) Management strategy based on concerted monitoring to optimize the implementation of public policies. Tarapoto, Peru. (doctoral thesis) César Vallejo University. $\quad$ Recovered from http://repositorio.ucv.edu.pe/handle/20.500.12692/38039?locale-attribute=en.

Van Horne, J. C. (2002). Administración Financiera (11ava edición), México. Pearson Educación, México.

Vargas, S. (2018). Sistemas administrativos y gestión por resultados en la Municipalidad Provincial de San Martín- 2018. Perú (Tesis de Doctorado) Universidad César vallejo. Recuperado de https://alicia.concytec.gob.pe/vufind/Record/UCVV_0b449707635283c6f9e421da63 64333b/Details. 